\title{
Contribución al estudio de las causas de depreciación y oscilación del valor de la moneda en Chile.
}

\section{I.-EL BALANCE DE PAGOS ENTRE DOS PAISES CON MONEDA DE ORO}

\footnotetext{
En las líneas siguientes denominaremos:

A). Suma de fodos los pagos y salidas de dinero al exterior.

B). Suma de fodos los pagos y entradas de dinero en el país.

S). El saldo positivo o negativo que se obfiene de los dos sumandos anferiores.
}

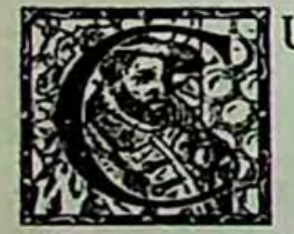

UANDO en un país existe moneda de oro y también existe en los demás países, y se tiene, además, la libre importación y exportación del oro, no se presentan realmente los fenómenos de cambio. Cuando el valor de (S) es positivo, entra el oro al país. Cuando (S) es negativo. sale oro del país. El cambio externo se transforma en cambio interno. La moneda no varía con el cambio, pero varía en su poder adquisitivo interno. Si durante varios años $(\mathrm{S})$ es negativo, sale oro del país; se restringe la circulación monetaria; se reduce el crédito; aumenta el tipo de interés; crece el valor relativo del oro y disminuye, también relativamente, el costo de la vida. La demanda por los depósitos se acumula en los Bancos y se produce la inevitable crisis monetaria y financiera.

Por la inversa, si durante varios años (S) es positivo, entra oro en el país; aumenta la circulación; disminuye el tipo de interés; se facilitan los créditos; se produce el boom en los negocios; el oro disminuye en su valor relativo y la 
vida, también relativamente, encarece. Los salarios aumentan y la producción se dificulta. La crisis se produce de nuevo por falta de exportación en relación con los nuevos costos.

El valor de (S) puede ser igual a cero, en correspondencia con igualdad entre créditos y débitos. En tal caso, no habrá fluctuaciones monetarias y el estandard, de oro permanecerá invariable.

Pero es lo común que (S) sea, alternativamente, positivo y negativo. Es decir. siempre habrá fluctuaciones en el valor de oro en el país. En tal caso, no habrá variaciones del cambio interno, pero habrá variaciones en el valor adquisitivo interno. O sea, el sstandard, de oro no es el sistema monetario perfecto. Carece de la elasticidad necesaria para anular o regular las perfurbaciones económicas.

\section{II.-EL BALANCE DE PAGOS ENTRE UN PAIS CON MONEDA INESTABLE Y UN PAIS CON MONEDA ESTABLE}

No he considerado otros casos de cambio internacional como, entre varios. el que relaciona dos paises, ambos de moneda inestable para entrar de lleno a la situación ofrecida por el problema nuestro.

Hemos visto que, para el país con moneda de oro o moneda estable, no existen problemas de cambio internacional (salvo el limite reducido del egold points). Sólo interesa, por consiguiente, analizar la situación del país que ofrece una moneda de carácter inestable.

Interviene en este caso el signo del valor (S). designación atribuída al Saldo del Balance de Pagos Internacionales.

$\mathrm{Si}(\mathrm{S})$ es negativo, es necesario cancelar con la moneda corriente ese valor. En este caso. (S) pertenece a poseedores extranjeros y es necesario adquirir. para la cancelación de (S), monedas extranjeras, o, generalmente, letras. Cualquiera que sea el procedimiento de pago, equivale a adquirir oro con billete. o sea, a despreciar el billete. Por consiguiente, mientras (S) sea negativo habrá depreciación persistente de la moneda.

Si $(S)$ es positivo, el valor de (S) pertenece a acreedores nacionales y su cancelación equivale, en definitiva, a adquirir billetes nacionales con oro o letras extranjeras. Naturalmente, el billete se valoriza. Si (S) fuera siempre positivo, la moneda billete continuaria continuamente valorizándose, siempre que el volumen de billetes en circulación no aumentase en cantidad. Pero los valores de (S) no pueden ser siempre negativos ni positivos. Son alternativamente positivos y negativos, y ello provoca alteraciones positivas y negativas del cambio externo.

\section{III.-CAUSAS DE DESVALORIZACION AJENAS AL BALANCE DE PAGOS}

Pero la moneda inestable. por su origen. presenta causas de desvalorización que actúan simultáneamente con el saldo (S) del Balance de Pagos. 
En realidad, como ya lo hemos dicho, el más elemental sentido común advierte que $(S)$ no puede ser siempre positivo, ni siempre negativo. O sea, que un país no puede permanentemente gastar más de lo que recibe, ni recibir más de lo que entrega. Ello sería contrario a la ley general del equilibrio económico que asegura la vida comercial a las naciones. Puede un país, en un escaso número de años, exportar más de lo que importa. Pero pronto la riqueza acumulada lo impulsará a efectuar nuevas importaciones o gastos de dinero al exterior. Por la inversa. si un pais mantuviera, durante varios años consecutivos, un saldo desfavorable en su balance, primero perdería los saldos anteriores favorables de su Balance de Pagos y. en seguida, necesitaría ir realizando su riqueza pública y privada. Por otra parte, la misma fluctuación del cambio se encarga de reducir las importaciones y los gastos de dinero en países extranjeros.

En resumen, puede decirse que, en un cierto periodo, la suma de los (S) positivos debe ser igual a la suma de los $(S)$ negativos. Esto equivale a decir que. dentro de ese mismo periodo. el término medio del cambio internacional debería permanecer constante.
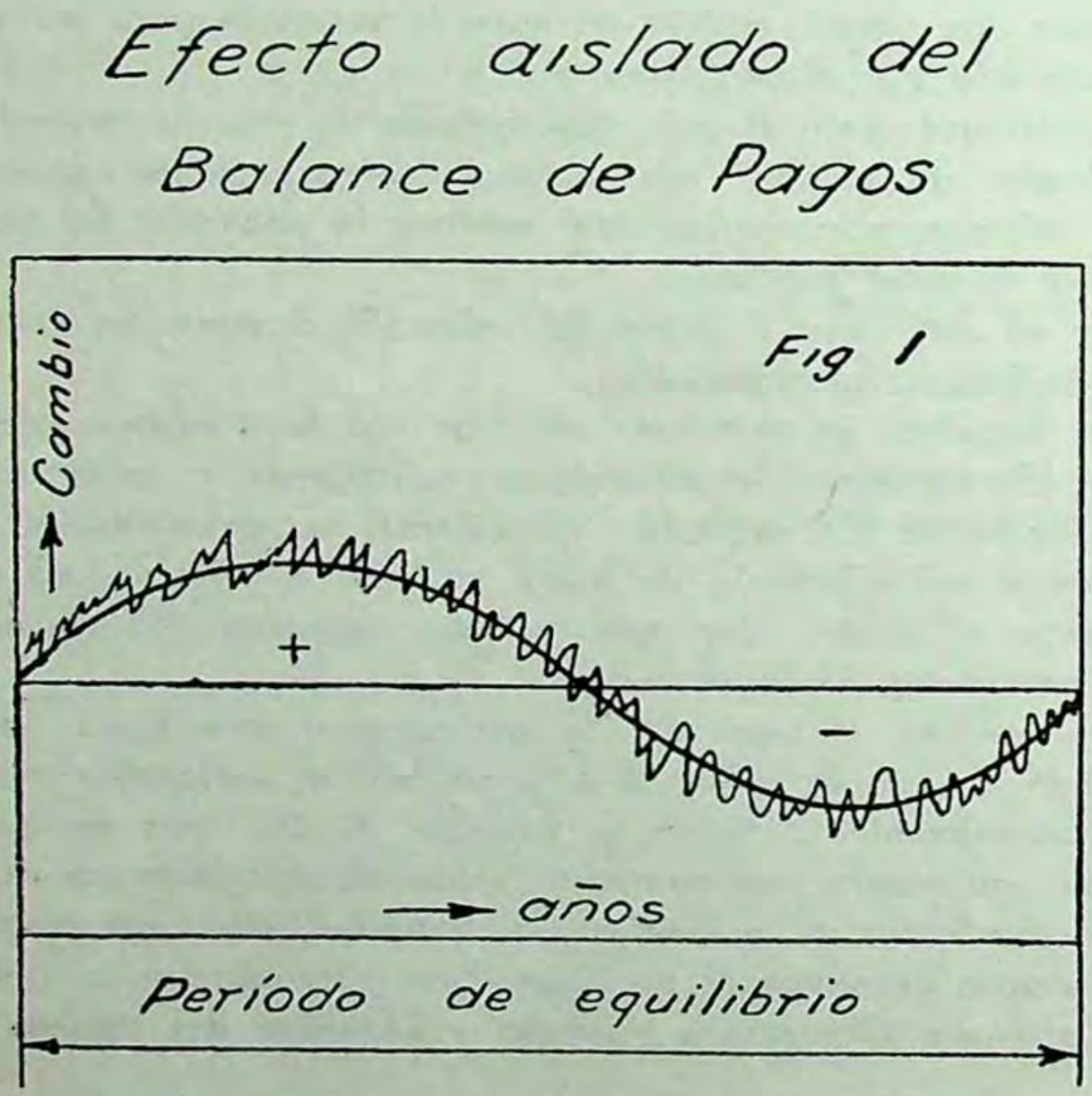

Representada analíticamente en la fig. 1 , la curva del cambio, ella corresponderá a una curva sinusoidal con su eje paralelo a la línea de los años. El saldo del Balance de Pagos Internacionales explica, en consecuencia, la oscilación del valor de la moneda, pero en su depreciación continua. 
Depreciación y oscilación del valor de la moneda en Chile.

\section{IV.-CAUSAS DE DESVALORIZACIÓN AJENAS AL BALANCE DE PAGOS. (CONTINUACIÓN).-EL CASO DE CHILE}

Si se traslada a un gráfico la curva de la depreciación de la moneda en Chile, y si se toma el periodo de años que corresponde a la caida de los 43

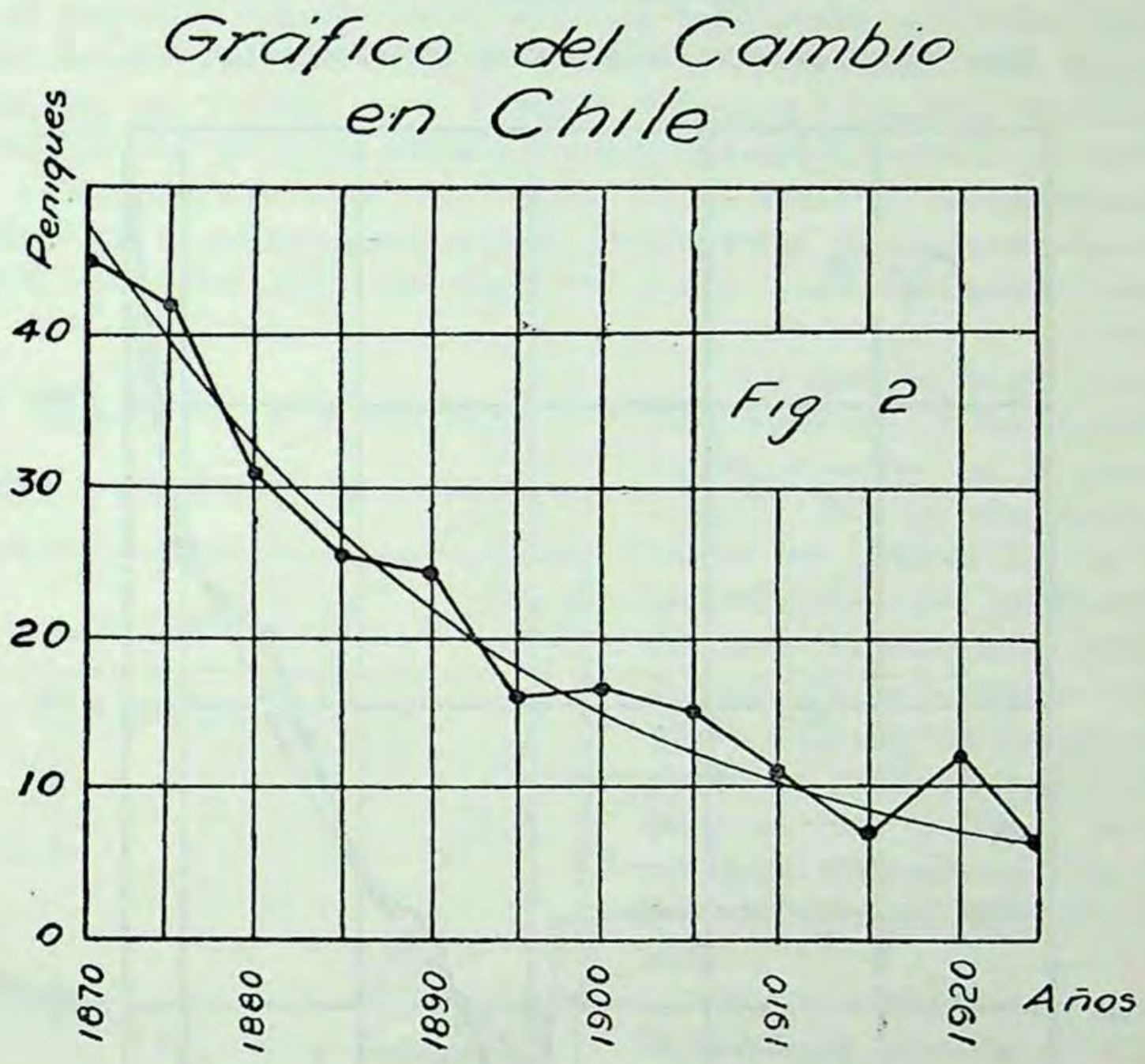

a los 6 peniques. (1872 a 1922). Fig. 2, se pueden observar dos caracteristicas generales bien precisas:

1. Que la curva sigue oscilaciones alternativas positivas y negativas.

2. Que el eje de la curva sigue una inclinación general que empieza en 47 peniques y termina en 6 peniques.

La primera característica es fácil de explicar por los signos alternativos de (S), saldo del Balance de Pagos, los cuales han actuado en cada momento por el hecho de ejercer sus efectos sobre moneda de papel no convertible.

La segunda característica no es explicable por el Balance de Pagos. En efecto. la curva de la fig. 2, abarca un periodo de medio siglo, en el cual no es concebible suponer que el pais se haya estado empobreciendo por medio de la cancelación de saldos continuamente desfavorables del Balance de Pagos. Si eso hu- 
biese acontecido, no existiría hoy ningún capital público ni privado en manos de poseedores nacionales.

Es aqui donde conviene introducir la causa verdadera de la depreciación de la moneda, causa que se debe superponer a la del Balance de Pagos, y que no

Curva del papel monedo en

circulación

en millones monedá corriente.

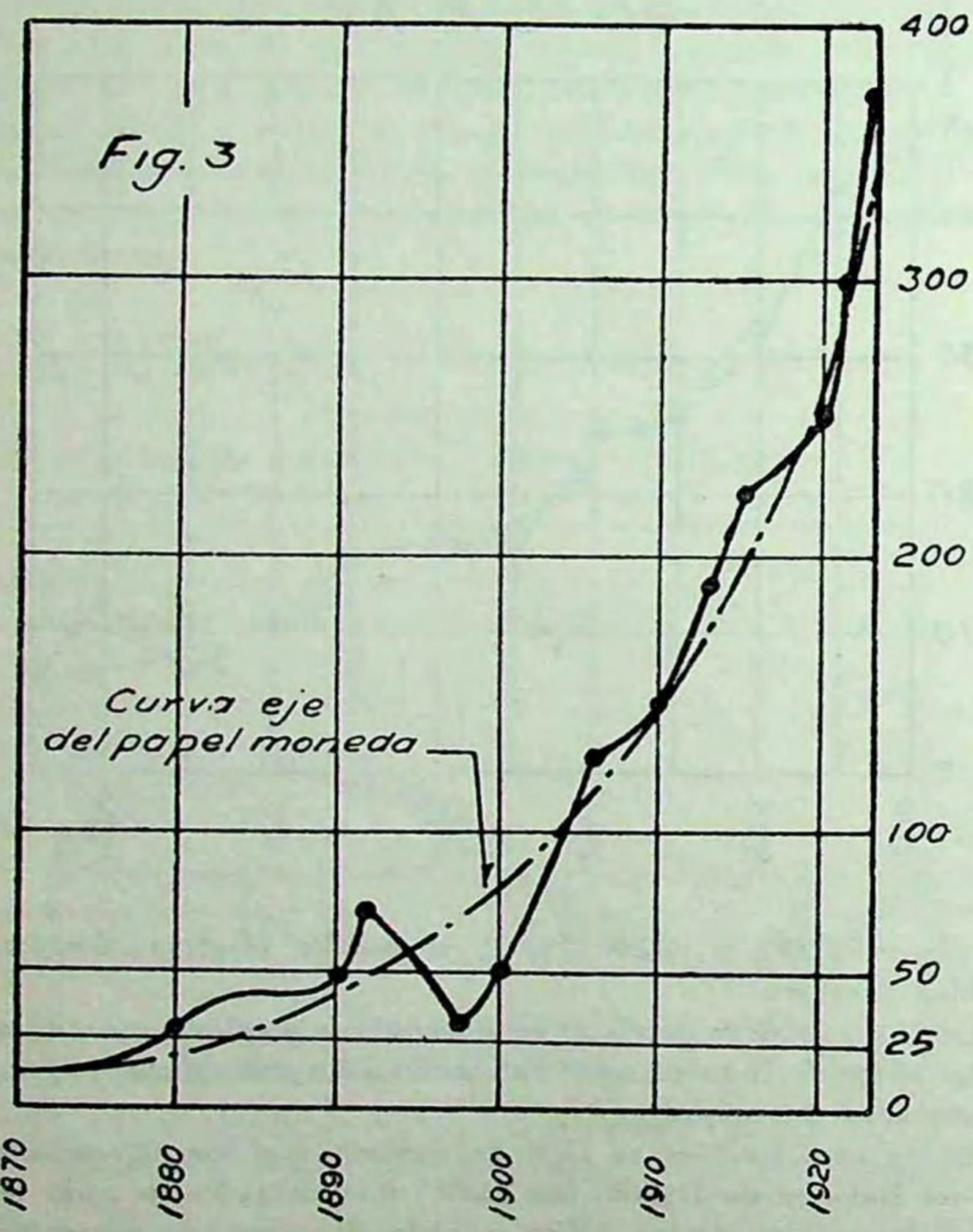

siempre se considera en su efecto verdadero sobre el cambio. Dicha causa, como se ha comprobado, no puede ser otra que el papel moneda de curso forzoso.

El papel moneda de curso forzoso no es moneda y no vale sino por el curso forzoso que le impone la ley. Su efecto no es crear moneda, no es crear 
valores, no es crear oro. En la teoría y en la práctica, equivale a subdividir la moneda existente, ya que la aumenta en cantidad y no en valor. Ahora bien, una moneda que se subdivide baja de valor en cantidad proporcional a esa misma subdivición. Por ejemplo: en un pais circulan cien millones de monedas de oro de valor de un dólar cada una. Todas las transacciones internas y externas del país se efectúan con esos cien millones. Pero el Gobierno, por cualquier motivo, emite cien millones de papel moneda sin convertirlos en oro. En tal caso. habrá en el país 200 millones para atender a necesidades que valen cien millones. Aparte de eso, los 200 millones no valen, ante el exterior. más que los 100 millones en oro que existian en el momento anterior a la emisión. Puede todavía el Gobierno guardar en arcas fiscales cualquiera reserva metálica de oro. Pero. como en el hecho, esa reserva no circula, ni el billete es convertible con esa reserva, ella hace el mismo papel que si, en definitiva, no existiera. En resumen, los 200 millones valen 100 millones oro, o sea, cincuenta centavos oro cada peso.
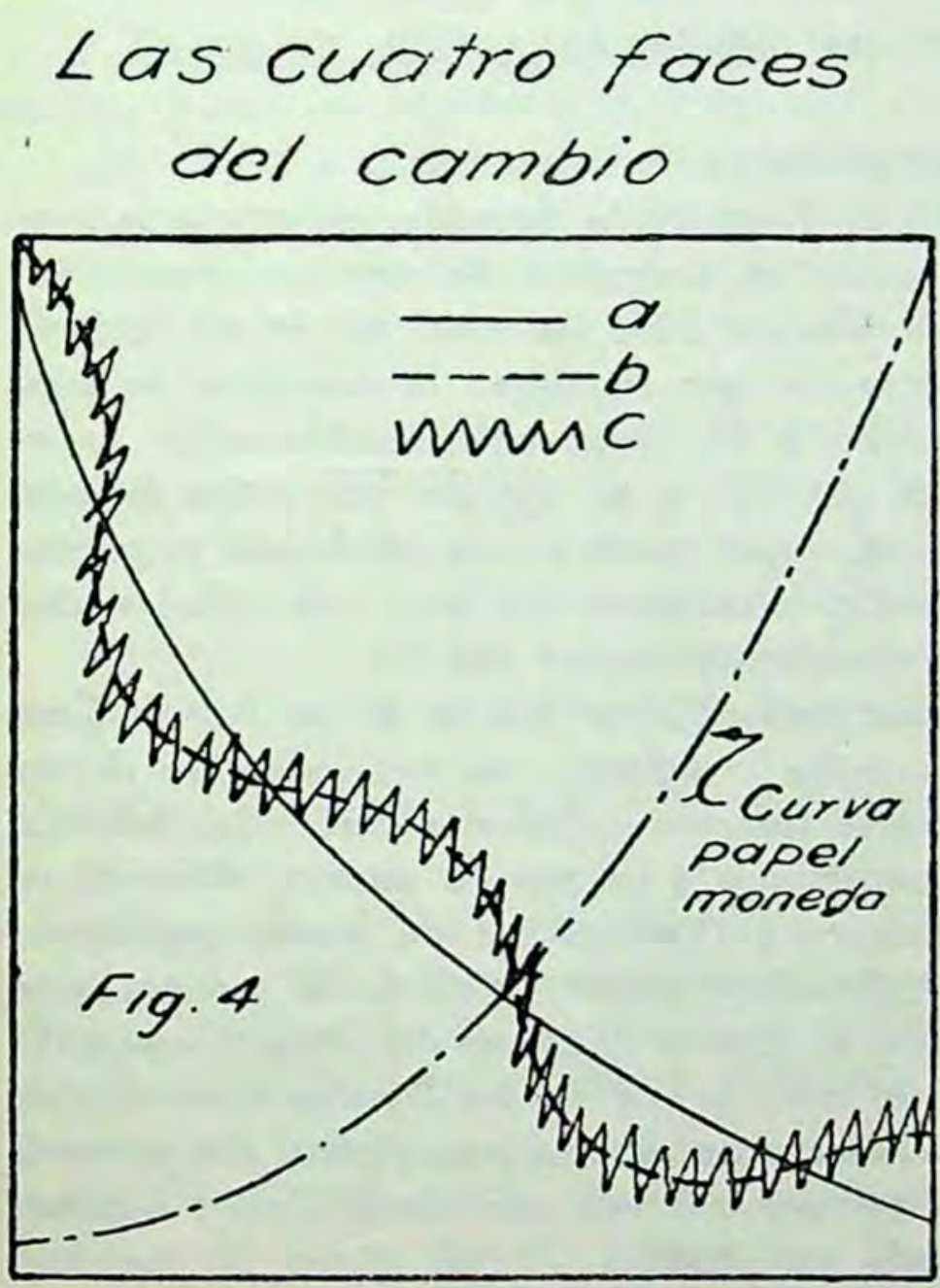

La emisión de papel en Chile ha seguido una curva correlativa con la depreciación en el valor de la moneda. Ello se demuestra observando los gráficos 2 y 3. La circulación monetaria inconvertible empieza con 12 millones en 1879 (antes existían billetes bancarios por valor de 14 millones, los cuales subsistieron hasta 1897) alcanza a $302 \mathrm{mi-}$ llones en 1920 y llegará en 1924 a cerca de 400 millones, con la emisión proyectada de Vales del Tesoro...

Estos 400 millones, agregados al circulante existente en Chile, no valen nada, absolutamente nada. El Fondo de Conversión tampoco les atribuye valor real. pues ese fondo no representa, en el hecho, función monetaria alguna. En resumen, el caso nuestro equivale prácticamente al de una emisión inconvertible con incrementos sucesivos.

\section{V.-LAS TRES FASES DEL CAMBIO}

La fig. 4 resume, en lineas teóricas generales, los hechos establecidos en las figs. 1, 2 y 3 , explicados en los títulos II. III y IV de este estudio. 
Ella permite apreciar la existencia de tres efectos graduales y sucesivos sobre el cambio. Ellos son:

Linea a.-La depreciación continua de la moneda, provocada por el incremento también continuo del volumen de papel moneda inconvertible.

Linea b.-Las oscilacioncs periódicas y alternativas en más y en menos sobre la línea general de depreciación, provocadas por la existencia de saldos alternativos en nuestro Balance de Pagos Internacionales.

Linea c.-Las oscilaciones circunstanciales provocadas dentro de un mismo periodo por razones de especulación o por desigual repartición del saldo del Balance de Pagos dentro de un periodo.

La existencia de estas tres causas y sus efectos puede sensiblemente apreciarse formando la curva de valores del cambio en Chile a partir de la fecha de existencia del papel moneda inconvertible.

La fig. 2, aunque dibujada a escala reducida y con valores de cinco en cinco años, permite comprobar, con la realidad de los hechos, las teorías enunciadas.

\section{VI.-LAS TRES SOLUCIONES AL PROBLEMA DEL CAMBIO}

Para tres causas deben existir, naturalmente, tres soluciones.

Causa a.-Para evitar la oscilación continua de la moneda, es necesario convertirla a oro. Para evitar su depreciación, es necesario detener sus emisiones. En el tifulo $I$ hemos advertido que la circulación libre del oro no es un sistema monetario que ofrezca la elasticidad necesaria que requieren la industria, la producción y los negocios. Asimismo, no ofrece a las organizaciones bancarias expedientes de emergencia. La solución más general, y la seguida por todos los países-incluso Estados Unidos e Inglaterra,-que poseen una verdadera organización bancaria y monetaria, es la existencia simultánea del oro y de billetes, con mayores o menores facilidades para la circulación interna del oro.

La conversión se efectúa así, permanentemente, por medio de un Banco Central. El sistema permite aumentar la moneda circulante, sin necesidad de depreciarla. Basta para ello mantener una cierta relación entre el encaje y los billetes. Esa proporción depende de diversas condiciones y es, por lo mismo, diferente en la mayoría de los paises. Así, en Inglaterra y Francia, era de ciento por ciento antes de la guerra. En Alemania, de treinta por ciento antes de la guerra, y se consulta el mismo treinta por ciento en el Banco Central del Plan Dawes. El Banco de la República en Colombia, el más nuevo de los Bancos Centrales en servicio en Sud-América, ha fijado una reserva de sesenta por ciento. En general. la experiencia no recomienda un limite inferior a treinta por ciento, y un máximo superior al ciento por ciento es estimado innecesario.

Causa b.-Dara evitar balances de Pagos negativos, los cuales una vez establecido el Banco Central, no alterarian el cambio, pero provocarian salida de oro o la obtención de créditos externos, es necesario aumentar la producción. lavorecer la exportación y restringir la importación. Es aqui donde debe intervenir la fórmula de sproducir más y consumir menos. A esto debe agregarse que, pre- 
cisamente, lo que más reclaman nuestras actividades productoras e industriales es una moneda estable que les permita asegurar sus créditos y precios.

Causa c.-Las oscilaciones circunstanciales de nuestro cambio dependen de la variación irregular en las cantidades de letras pedidas u ofrecidas o, lo que es lo mismo, de la distribución irregular, dentro de un periodo, del saldo del Balance de Pagos. Esta causa queda de hecho eliminada por la intervención del Banco Central.

\section{VII.-EL BANCO CENTRAL. CONDICIONES PARA SU ESTABLECIMIENTO}

Las tres causas que provocan las oscilaciones del cambio pueden ser evitadas por el Banco Central, siempre que las condiciones para su establecimiento sean favorables.

Esas condiciones son:

1. Que el Banco Central pueda disponer de un estock, de oro suficiente para recoger la emisión de billetes fiscales en circulación y reemplazarlos por billetes bancarios convertibles bajo la responsabilidad del Banco.

$2 .^{\circ}$ Que el valor real de la moneda en el momento de recogerse la emisión no corresponda a un valor circunstancialmente elevado que obligue en seguida, para mantenerlo, a una salida de oro.

3. Que el derecho de emisión sea abandonado por el Gobierno en favor del Banco Central.

4. Que los préstamos del Banco Central al Gobierno sean limitados a un máximo compatibles con la seguridad del Banco. condición que equivale a la existencia de presupuestos financiados o, en su defecto, de un buen crédito fiscal externo al cual acudir en caso de emergencia.

5. Que el sstock, de oro del Banco Central sea suficiente para resistir un periodo de Balance de Pagos Internacionales desfavorable.

\section{VIII._EL BANCO CENTRAL. POSIBILIDADES DE ESTABLECIMIENTO}

Respecto de la condición $10^{2}$ - La actual emisión, que sumará luego aproxi_ madamente, 350 millones en billetes, necesitará un encaje de oro variable entre $30 \%$ y $100 \%$. Si nos ponemos en el caso más desfavorable. o sea, en el del máximo superior, se necesitaría un encaje de 350 millones de oro. A razón de 7 peniques por peso, el Fondo de Conversión de 114 millones oro de 18 que guarda el Estado en la Casa de Moneda permitiria formar un sstock, de 294 millones de pesos oro de 7 peniques. La relación de conversión sería entonces de $85 \%$. Al no realizarse la emisión proyectada de 110 millones en billetespropuesta para saldar el actual déficit fiscal-la relación de conversión pasaría de $100 \%$. Finalmente, si, como se ha hecho en los nuevos Bancos Centrales de Colombia y del Perú, - organizados al estilo del Federal Reserve Bank de los EE. UU.-se pide también la contribución de los bancos privados y del público 
para la formación del capital en oro del Banco Central, se puede asegurar que disponemos de oro en exceso para la formación del Banco y realizar el . Gold Exchange Standard.

Respecto de la condición $2 .^{3}$.-El valor real de la moneda-entre 7 y 6 peniques-es el más bajo alcanzado, en un periodo apreciable. por la curva del cambio en Chile. O sea, es éste el mejor momento para efectuar la conversión.

Respecto de la condición 3."-Debe ser ésta la condición primera por establecer en la ley Orgánica del Banco Central.

Respecto de la condición $4 .^{2}$-Esta condición también debe establecerse en la ley Orgánica. La estabilidad de la Hacienda Pública, por otra parte, aparece ya prácticamente fundada en las nuevas leyes tributarias. Por lo demás, el último balance no señala incremento apreciable en el déficit de 110 millones en billetes arrastrado por varios años.

Respecto de la condición 5. ${ }^{\text {- }}$-Determinar la salida de oro (o la garantía contra un crédito en Bancos Extranjeros) necesaria para no desvalorizar el cambio en caso de un Balance de Pagos desfavorable, es un problema de solución incierta. Nunca se puede saber en cuanto difiere el Balance de Pagos del Balance Aduanero. Pero se puede aceptar el paralelismo de las curvas respectivas y la influencia predominante del Balance Aduanero respecto de los demás factores que completan el Balance de Pagos Internacionales.

Analizando la situación de nuestro Balance Aduanero desde 1913 a 1923 (fig. 5), puede observarse que la diferencia mayor entre los saldos corresponde a

\section{Saldos del Balance Aduanero}

en millones de pesos oro de $18 \mathrm{~d}$.

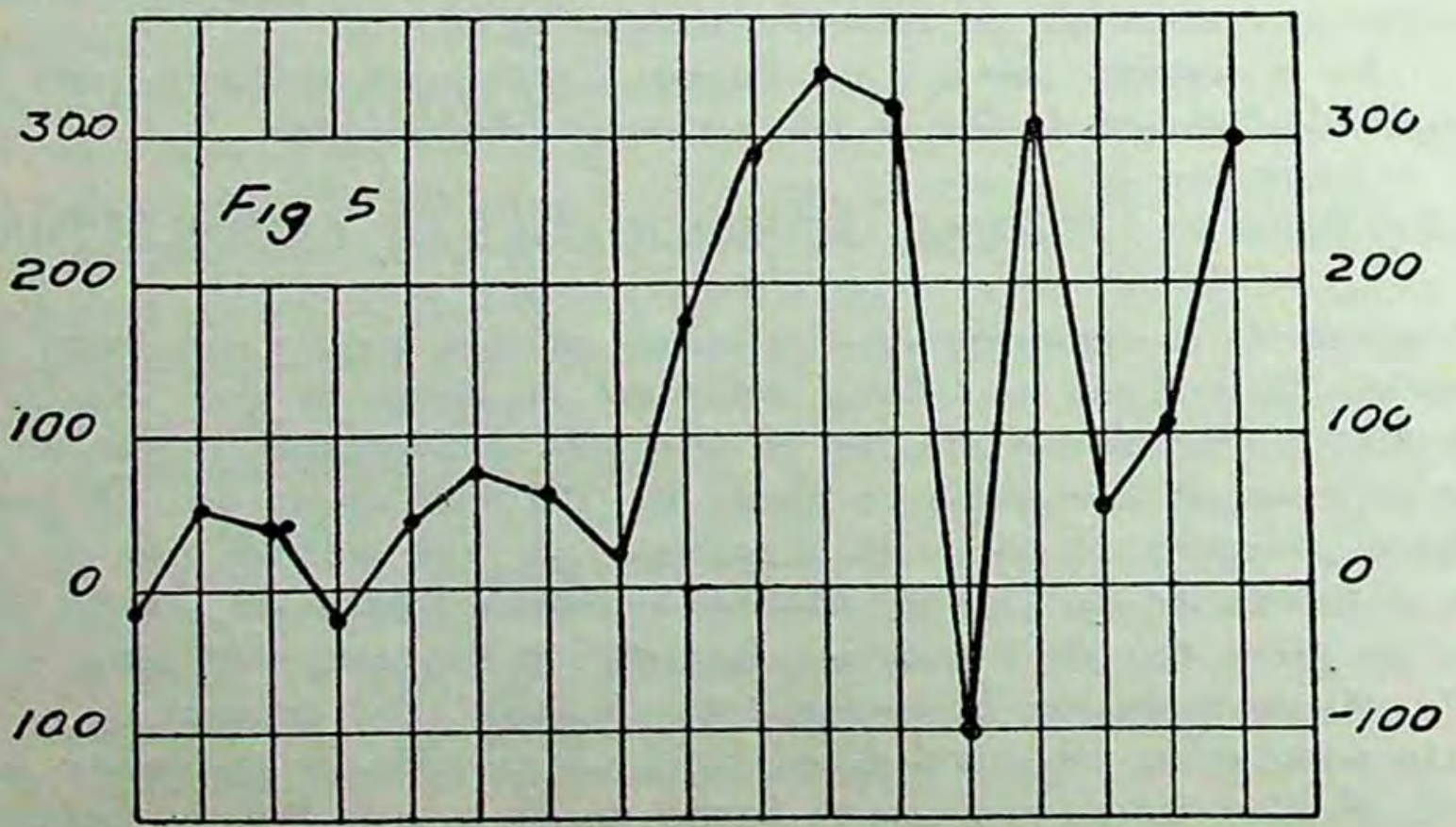

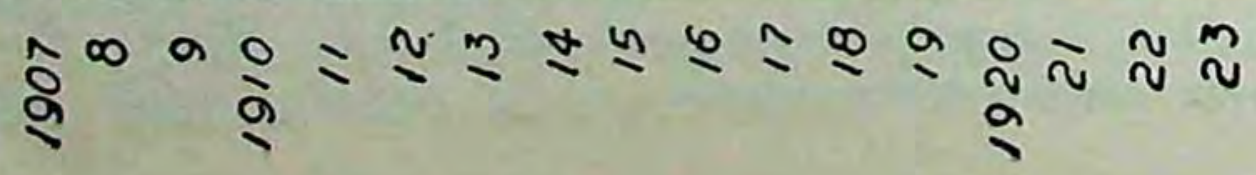


la crisis salitrera de 1919 y 1921. en relación con una oportunidad que talvez nunca vuelva a repetirse en la historia económica mundial. La diferencia de saldos entre el año 18 y el 19 pasó de 400 millones oro de 18 peniques y su efecto sobre el cambio no habría podido ser soportado por ningún Banco Central establecido justamente el año anterior a la misma crisis.

Pero ese mismo ejemplo nos lleva a comprobar la periodicidad de las oscilaciones alternativas del Balance Aduanero. En efecto. si suponemos el Banco Central fundado el año 13, antes de soportar la crisis del año 19. habría recibido los efectos favorables de los años 15, 16, 17 y 18, los cuales habrian permitido afrontar la crisis ede compensación, producida al terminar el periodo de ebooms.

De la misma manera, el gráfico señala crecimientos favorables del saldo aduanero a partir de 1922. los cuales tienen el carácter de compensación de la crisis de 1921. Finalmente, es lógico suponer que esos saldos favorables se acentúen en los años próximos, en primer término. por la reducción de las importaciones que seguirá al alza de los aranceles aduaneros y en segundo, por la misma depreciación actual de la moneda que, de hecho, impide la salida de dinero al exterior, ya sea en concepto de importaciones o de gastos invisibles.

La situación actual de nuestro comercio exterior aparece, pues, favorable para la formación del Banco Central.

Ahora, supuesta, alcanzado la normalidad comercial de los años anteriores a la guerra, y adoptando como ejemplo el período comprendido entre los años 7 al 13. puede observarse el gráfico citado que los saldos aduaneros sólo ocasionalmente presentan caidas mayores de 50 millones oro de 18 peniques, y que. normalmente, las diferencias en los saldos fluclúan entre diez y veinte millones oro. cantidad susceptible de ser soportada por cualquier Banco Central, sobre todo si se toma en consideración que esas caidas son siempre en compensación de acumulaciones favorables anteriores.

El gráfico de la fig. 5 señala una observación importante. Ella es el mayor valor de los saldos aduaneros lavorables a partir de 1922 que en los años anteriores a 1913. Ello se debe a que, por el aumento del servicio de la Deuda Pública Externa y por el mayor servicio de capitales extranjeros invertidos en el pais, se requiere un mayor saldo aduanero lavorable para compensar las epartidas invisibles, de salidas de dinero al exterior. La necesidad de cubrir esas partidas de pagos sinvisibles, es lo que hace que, a pesar del saldo aduanero favorable de 300 millones de oro en 1923. el cambio se haya mantenido depreciado.

\section{CONCLUSION}

He tratado. en estas lineas, de demostrar que la obtención de Saldos Favorables en nuestro Balance de Pagos Internacionales no es todo para la solución de nuestro problema monetario. Tan importante como un Saldo Favorable es una moneda convertible y. por lo tanto, un Banco Central que se encargue de convertirla. Naturalmente, se trata del caso de un Banco Central que disponga de un estock, de oro suficiente para recoger las emisiones en vigencia de papel moneda 
de curso forzoso. Ello equivale a emplear nuestros fondos de conversión en el objeto a que realmente han sido destinados. La existencia simultánea de un Banco Central y de los Fondos de Conversión,- - sin correlación entre ambos, - no será. por consiguiente, la solución de nuestro problema monetario.

\author{
RAÚL SIMÓN.
}

(Jefe de la sección Finanzas y Estadisticas de los Ferrocarriles del Estado de Chile).

New York, 28 de Agosto de 1924. 